\title{
Mixed integer formulation for multiproduct maritime inventory routing problems
}

\author{
Syahrul Maulana*, Farida Hanum, Toni Bakhtiar \\ Division of Operations Research, Department of Mathematics, Bogor Agricultural \\ University, Kampus IPB Dramaga, Bogor 16680, INDONESIA \\ *Corresponding author: maulsyahrulana17@gmail.com
}

\begin{abstract}
Companies that want to market their products to the outer islands need a largecapacity transportation mode that can distribute the product to every place. Other important consideration is the cost of the company must be efficient. The most commonly used by transportation logistics company is the mode of sea transportation. This paper presents an optimization model for determining vessel travel routes by meeting demand and paying attention to inventory levels at each ports so that the company's costs are minimum. The problem is known as the maritime inventory routing problem (MIRP) and the delivered product can be just one type (single product) or it can be multiproduct. MIRP models are formulated using integer linear programming and numerically solved by the aid of MiniZinc IDE 2.1.5.
\end{abstract}

\section{Introduction}

It is forecasted by UNCTAD (2017) [10] that world seaborne trade to increase by 2.8 per cent in 2017, with total volumes reaching 10.6 billion tons. It is also projected that expansion continues with volumes growing at an estimated compound annual growth rate of 3.2 per cent between 2017 and 2022. Cargo flows are expected to expand across all segments, with containerized and major dry bulk commodities trades recording the fastest growth. These facts suggest an effective maritime transportation of utmost importance.

In maritime transportation, huge quantities of products are usually loaded and unloaded at production and consumption ports. In this situation, the loading process, inventory and transportation are time consuming and costly (Christiansen et al., 2011) [6]. Thus, the routing and scheduling of the ships as well as the inventory management should be planned simultaneously. The resulting problem is called a maritime inventory routing problem (MIRP) (Agra, et al., 2018; Song and Furman, 2013) [1][9]. Optimization in maritime inventory routing is a well-established field of research in transportation planning with growing amount of research in the literature in various applications during the last decade (Christiansen and Fagerholt, 2009) [4].

This paper extends the work of Song and Furman (2013) into a multiproduct MIRP, where the model is proposed and formulated in a mixed integer programming. An optimization model for determining ships travel routes by meeting demand and paying attention to inventory levels at each place so that the company's costs are minimum is considered

\section{Related literature}

Some researchers have explored the issue of MIRP, as Christiansen and Nygreen (1998) [5] did which examined the distribution of a single product on several production ports with a constant production 
rate and limited storage capacity taking into account travel time, the model was solved by column generation techniques. Christiansen (1999) [3] examined the problem of maritime inventory routing about the combination of inventory problems and the determination of ship routes with time constraints, and the model was solved using the Dantzig-Wolfe decomposition approach. Christiansen and Fagerholt (2009) [4] describe the usual MIRP as the transportation of one product produced in a production port and sent to a consumption port. Al-Khayal and Hwang (2007) [2] also explain the varied aspects of maritime transportation which formulate the problem of nonlinear programs into linear problems with special structures that minimize ship travel costs in loading and delivering large quantities of liquid / liquid products

\section{Problem description}

Suppose there are several production ports $J^{P}$ that will produce products sent to the port of consumption $J^{C}$ in a certain period $T$. Each port has an initial inventory of products and has a minimum and maximum inventory limit. Travel time between ports is assumed in a day. Ships available to deliver products have different capacities and cost. A journey begins when the ship is empty and ends when it is empty again. Based on the depth level of the seawater in the port, the ships that come and go have product draft limits. Settlement of distribution and inventory problems requires costs which consist of the cost of renting a vessel, overloading which exceeds the minimum part cargo vessel as well as the cost of travel to the destination port and demurrage costs then subtracting the netback multiplied by the number of delivered products. The purpose of this problem is to find the optimal route with the minimum cost of the ship trip by taking into account certain capacities, constraints and limitations. Examples of illustrated ship trips can be seen in figure 1 .

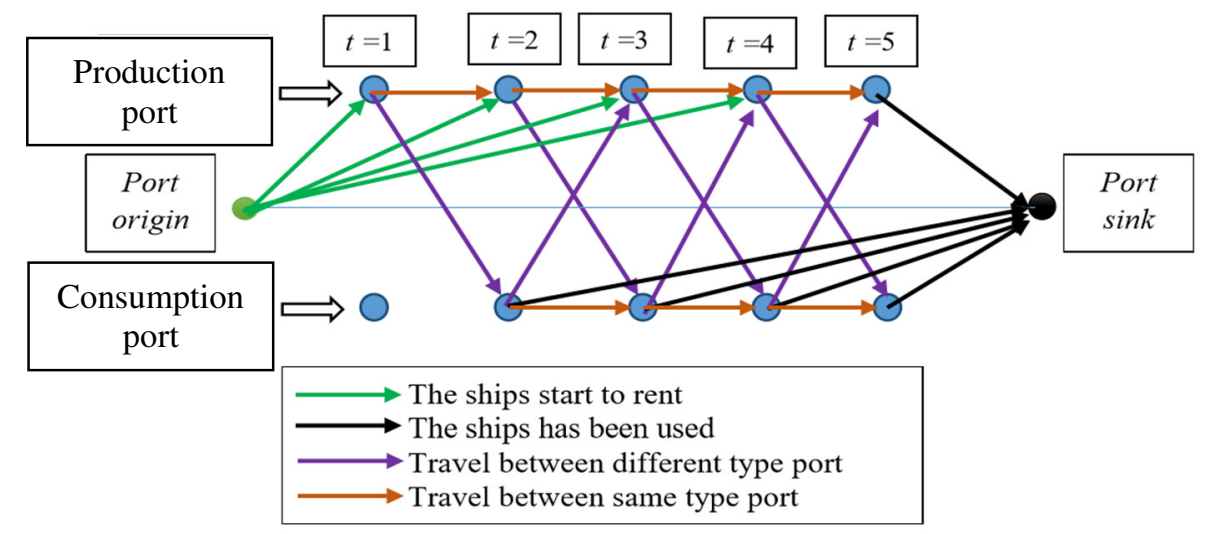

Figure 1. Illustration of a ship trip

MIRP problems can be described by a time-space network, which consists of a set of nodes and a set of arcs. Nodes represent possible inter-port trips that can be written as $(j, t)$ with $j$ declaring a port and $t$ declaring a specified time. The node $(j, t)$ states the ship's visit to port $j$ at time $t$. The node set is divided by each ship and each ship has its own set of arc. The set of nodes consists of the origin node $(0,0)$, sink node or end node $(0, T+1)$, and the set of regular nodes, namely nodes other than the original node and end node. Arc states the possibility of a trip by a ship that can be written as $(v,(j, t),(k, t+1))$ with $v$ denotes the ship and $j$ is the initial port at time $t$ and $k$ is the destination port that reaches the time $t+1$ (Song and Furman, 2013) [9].

This problem is defined as follows. Given:

- a discrete model with units of time in days,

- horizon/planning period along $T$,

- the number of products / goods produced or consumed per day on each port is known,

- single shipped products for each port,

- each port has a product limit at the time of entry or exit port and has a minimum limit and maximum product inventory for each time, 
- only one ship can load or discharge in one day,

- total vessel visits for loading or discharging to all ports are limited,

- travel time between ports is only one day.

This paper modifies the model by Song and Furman (2013) [9], where the optimal schedule for routing a heterogeneous vessels to send a single bulk product while maintaining all capacities and inventory levels at each ports was studied. This paper developed a multiproduct MIRP problem which involves two types of products.

\section{Model}

\subsection{Notation}

The following sets, parameters, and variables are used throughout the paper.

Sets

$V \quad$ set of all vessels with index $v$ where $V=V^{C} \cup V \backslash V^{C}$

$V^{C} \quad$ set of vessels that hired

$V \backslash V^{C} \quad$ set of vessel that unhired

$J \quad$ set of all ports wit index $j$ where $J=J^{P} \cup J^{C}$

$J^{P} \quad$ set of production port

$J^{C} \quad$ set of consumption port

$N \quad$ set of all node with index $n$

$N^{R} \quad$ set of regular node

$A \quad$ set of all arc with index $a$ where $\bigcup_{v \in V} A_{v}$

$A_{v} \quad$ set of arcs belonging to vessel $v$

$\delta^{+}(n) \quad$ set of arcs that have node $n$ as their tail node

$\delta^{-}(n) \quad$ set of arcs that have node $n$ as their head node

$B \quad$ set types of product sent with index $b$

Parameters

$I P_{(j, b, 0)} \quad$ the initial amount of product type $b$ inventory level at production port

$I P_{(j, b, t)}^{\min } \quad$ minimum amount of product type $b$ in production port at time $t$

$I P_{(j, b, t)}^{\max } \quad$ maximum amount of product type $b$ in production port at time $t$

$I C_{(j, b, 0)} \quad$ the initial amount of product type $b$ inventory level at consumption port

$I C_{(k, b, t)}^{\min } \quad$ minimum amount of product type $b$ in comsumption port at time $t$

$I C_{(k, b, t)}^{\max } \quad$ maximum amount of product type $b$ in consumption port at time $t$

$b_{v b} \quad$ part cargo minimum for type $b$ in vessel $v$

$w_{v} \quad$ world scale multiplier for vessel $v$

$r_{v}^{o} \quad$ overage multiplier scale for vessel $v$

$D P_{v b j t}^{I} \quad$ draft limits which restrict the cargo capacity when entering production port

$D P_{v b j t}^{O} \quad$ draft limits which restrict the cargo capacity when exiting production port

$D C_{v b k t}^{I} \quad$ draft limits which restrict the cargo capacity when entering consumption port

$D C_{v b k t}^{o} \quad$ draft limits which restrict the cargo capacity when exiting consumption port

$c_{j k} \quad$ cost rate for traveling from port $j$ to port $k$

$C_{v b} \quad$ capacity in vessel $v$ for product type $b$

$c_{a} \quad$ the cost for using arc $a$

$p_{(b, j, t)} \quad$ the number of products $b$ produced by the production port $j$ from time $t-1$ to time $t$

$d_{(b, k, t)} \quad$ the number of products $b$ consumed by the consumption port $k$ from time $t-1$ to time $t$

$f p_{b j}^{\min } \quad$ minimum number of product type $b$ that loaded on production port

$f p_{b j}^{\max } \quad$ maximum number of product type $b$ that loaded on production port

$f c_{b k}^{\min } \quad$ minimum number of product type $b$ that discharged on consumption port 


$\begin{array}{ll}f c_{b k}^{\max } & \text { maximum number of product type } b \text { that discharged on consumption port } \\ m_{j} & \text { maximum times to loads product } \\ m_{k} & \text { maximum times to discharge product } \\ \eta & \text { netback of each unit of product delivered by the ship. }\end{array}$

Binary Variables

$x_{a} \quad 1$ if vessel $v$ use arc $a, 0$ otherwise

$y_{v b j t} \quad 1$ if vessel $v$ load product $b, 0$ otherwise

$z_{v b k t} \quad 1$ if vessel $v$ discharge product $b, 0$ otherwise

Continuous Variables

$f p_{v b j t} \quad$ number of product loaded

$f c_{v b k t} \quad$ number of product discharged

$I P_{(b, j, t)}$ inventory levels in production port

$I C_{(b, k, t)}$ inventory levels in consumption port

$I V_{v b t} \quad$ number of product in vessel $v$

$o_{v b a} \quad$ overage products in vessel $v$

\subsection{Model formulation}

The objective function of this problem is to minimize distribution costs which consist of ship usage costs and ship travel cost for overage product then reduced by netback for one ton of products delivered:

$\min \sum_{a \in A} c_{a} x_{a}+\sum_{v \in V} \sum_{a \in A} \sum_{b \in B} w_{v} r_{v}^{o} c_{j k} o_{v b a}-\sum_{v \in V} \sum_{k \in J^{c}} \sum_{t \in\{1,2, \ldots, T\}} \sum_{b \in B} \eta f c_{v b k t}$.

The constraints below describe a time-space network based formulation with flow conservation constraint:

$\sum_{a \in A_{v} ; a \in \delta^{+}(n)} x_{a}-\sum_{a \in A_{v} ; a \in \delta^{-}(n)} x_{a}=0, \quad \forall v \in V, \forall n \in N^{R}$,

$\sum_{a \in A_{v} ; a \in \delta^{+}(0,0)} x_{a}=1, \quad \forall v \in V$,

$\sum_{a \in A_{v} ; a \in \delta^{+}(0, T+1)} x_{a}=1, \quad \forall v \in V$.

The nest set of constraints explain about inventory balance at each ports, namely production ports and consumption ports:

$I P_{(b, j, t-1)}-\sum_{v \in V} f p_{v b j t}+p_{(b, j, t)}=I P_{(b, j, t)}, \quad \forall j \in J^{P}, \forall b \in B, \forall t \in\{1,2, \ldots, T\}$
$I C_{(b, k, t-1)}+\sum_{v \in V} f c_{v b k t}-d_{(b, k, t)}=I C_{(b, k, t)}, \quad \forall k \in J^{C}, \forall b \in B, \forall t \in\{1,2, \ldots, T\}$.

The next constraint is about inventory balance for products on the vessels are represented by the following expression:

$I V_{v b(t-1)}+\sum_{j \in J^{P}} f p_{v b j t}-\sum_{k \in J^{C}} f c_{v b k t}=I V_{v b t}, \quad \forall v \in V, \forall b \in B, \forall t \in\{1,2, \ldots, T\}$ 
The following set of constraints to ensure that a loading or discharging products by a vessel can occur only when the vessel is at that port.

$$
\begin{array}{ll}
y_{v b j t} \leq \sum_{a \in \delta^{-}(n)} x_{a}, & \forall n=(j, t) \in N^{R}, \forall j \in J^{P}, \forall v \in V, \forall b \in B, \forall a \in A_{v}, \\
z_{v b k t} \leq \sum_{a \in \delta^{-}(n)} x_{a}, & \forall n=(k, t) \in N^{R}, \forall k \in J^{C}, \forall v \in V, \forall b \in B, \forall a \in A_{v} .
\end{array}
$$

If the process of loading or discharging products is happen then the loading amount or the discharging amount has to be in between the port specific minimum and maximum amounts:

$$
\begin{aligned}
& f p_{b j}^{m i n} y_{v b j t} \leq f p_{v b j t} \leq f p_{b j}^{\max } y_{v b j t}, \quad \forall v \in V, \forall b \in B, \forall j \in J^{P}, \forall t \in\{1,2, \ldots, T\}, \\
& f c_{b k}^{m i n} z_{v b k t} \leq f c_{v b k t} \leq f c_{b k}^{m a x} z_{v b k t}, \forall v \in V, \forall b \in B, \forall k \in J^{C}, \forall t \in\{1,2, \ldots, T\} .
\end{aligned}
$$

In many other practical cases, berthing rules specify that only one vessel can stop for loading or discharging products at a port. If you want to change the numbers of vessels desired to carry out product for load or discharge more than one, then the right side of the constraints below can be adjusted appropriately:

$$
\begin{array}{ll}
\sum_{v \in V} y_{v b j t} \leq 1, & \forall b \in B, \forall j \in J^{P}, \forall t \in\{1,2, \ldots, T\}, \\
\sum_{v \in V} z_{v b k t} \leq 1, & \forall b \in B, \forall k \in J^{C}, \forall t \in\{1,2, \ldots, T\} .
\end{array}
$$

The next set of constraints is to uphold the assumption that total vessel visits for loading or discharging products are limited for the entire time the planning, we defined the limit total visits vessel by $m_{j}$ :

$\sum_{t \in\{1,2, \ldots, T\}} y_{v b j t} \leq m_{j}, \quad \forall v \in V, \forall b \in B, \forall j \in J^{P}$,

$\sum_{t \in\{1,2, \ldots, T\}} Z_{v b k t} \leq m_{k}, \quad \forall v \in V, \forall b \in B, \forall k \in J^{C}$.

The next set of constraints is to enforce the number of products on the vessel that will load in the production port or discharge in the consumption port so that when vessel enters or exits the port, it cannot exceed the specified limit due to water depth, but if the vessel does not load or discharge then the number of products only needs to below the vessel's capacity:

$$
\begin{array}{ll}
I V_{v b(t-1)} \leq D P_{v b j t}^{I}+\left(C_{v b}-D P_{v b j t}^{I}\right)\left(1-y_{v b j t}\right), & \forall v \in V, \forall b \in B, \forall j \in J^{P}, \forall t \in\{1,2, \ldots, T\}, \\
I V_{v b t} \leq D P_{v b j t}^{O}+\left(C_{v b}-D P_{v b j t}^{O}\right)\left(1-y_{v b j t}\right), & \forall v \in V, \forall b \in B, \forall j \in J^{P}, t \in\{1,2, \ldots, T\}, \\
I V_{v b t-1} \leq D C_{v b k t}^{I}+\left(C_{v b}-D C_{v b k t}^{I}\right)\left(1-z_{v b k t}\right), & \forall v \in V, \forall b \in B, \forall k \in J^{C}, \forall t \in\{1,2, \ldots, T\}, \\
I V_{v b t} \leq D C_{v b k t}^{O}+\left(C_{v b}-D C_{v b k t}^{O}\right)\left(1-Z_{v b k t}\right), & \forall v \in V, \forall b \in B, \forall k \in J^{C}, \forall t \in\{1,2, \ldots, T\} .
\end{array}
$$

The next constraint is for the overage number of products on a vessel when it exceeds the minimum part cargo of the vessel:

$$
o_{v b a} \geq\left(I V_{v b t}-b_{v b}\right)-\left(C_{v b}-b_{v b}\right)\left(1-x_{a}\right), \forall v \in V, \forall a \in A_{v} .
$$


The following set of constraints are for the inventory level of products in the production ports or the consumption ports must be in between the minimum limit and the maximum limit for the product at each ports:

$I P_{(j, b, t)}^{\min } \leq I P_{(b, j, t)} \leq I P_{(j, b, t)}^{\max }, \quad \forall b \in B, \forall j \in J^{\mathrm{P}}, \forall t \in\{1,2, \ldots, T\}$,

$I C_{(k, b, t)}^{\min } \leq I C_{(b, k, t)} \leq I C_{(k, b, t)}^{\max }, \forall b \in B, \quad \forall k \in J^{C}, \forall t \in\{1,2, \ldots, T\}$.

The next constraint is for ensure the number of products on a vessel does not exceed the capacity on the vessel:

$0 \leq I V_{v b t} \leq C_{v b}, \quad \forall v \in V, \forall b \in B, \forall t \in\{1,2, \ldots, T\}$

The next is for the limitation of products over the ship when it exceeds the minimum part cargo of the vessel:

$0 \leq o_{v b a} \leq C_{v b}-b_{v b}, \forall v \in V, \forall b \in B, \forall a \in A_{v}$

The final set of constraints are for non-negativity and for binary constraints:

$f p_{v b j t} \geq 0, f c_{v b k t} \geq 0, \quad \forall v \in V, \forall b \in B, \forall j \in J^{P}, \forall k \in J^{C}, \forall t \in\{1,2, \ldots, T\}$,

$x_{a} \in\{0,1\}, y_{v b j t} \in\{0,1\}, z_{v b k t} \in\{0,1\}, \forall v \in V, \forall b \in B, \forall j \in J^{P}, \forall k \in J^{C}, \forall a \in A_{v}, \forall t \in\{1,2, \ldots, T\}$.

\section{Results and discussion}

The optimization problem raised in this paper is slved numerically by MiniZinc, a programming language designed for optimization of problems with the decision variables in the form of real numbers. MiniZinc is designed with multiple solvers to produce optimal solutions by inputting the MiniZinc model and data files in the FlatZinc model. FlatZinc model consists of variable declarations and definitions of constraints and objective functions. Solvers contained in MiniZinc include Gecode (bundled), Gecode (Gist, bundled), Chuffed (bundled), COIN-OR CBC (bundled), Gurobi (bundled), G12 fd, G12 lazyfd, G12 MIP. MiniZinc can solve problems that are constrained easily because this software supports sets and arrays as well as types of decision variables that are diverse, namely integer, Boolean, set of integer. In addition, the advantages of this software are that there are declarations of many global constraints like all different, cumulative, regular tables, etc. which can be used to make the model easier. MiniZinc can also be used for different types of problems, namely satisfaction and optimization problems (Marriott et al. 2014) [7].

For an illustrative example, it is considered a simple routing problem of 4 ships connecting 5 production ports and 3 consumption ports and delivering 2 products namely Oil- 1 and Oil- 2 within 5 days of planning period. A number of parameters are utilized, such as the level of production and consumption in each port, including their initial levels and lower/upper bounds of inventory level, carrying capacity of ship, transportation and demurrage costs that can be seen in Tables 1-5. The problem was numerically solved by using software MiniZinc IDE 2.1.5 with solver COIN-OR CBC (bundled) under Windows 10 Pro operating system, processor intel/(R) core/(TM) i3-4150, CPU $3.50 \mathrm{GHz}, \mathrm{RAM} 4.00 \mathrm{~GB}$ which require 35 hours 11 minutes 43 seconds.

Table 1. Parameter values for each vessels

\begin{tabular}{ccccc}
\hline Notation & Vessel 1 & Vessel 2 & Vessel 3 & Vessel 4 \\
\hline$b_{v}$ & 2 & 3 & 4 & 5 \\
$C_{v}$ & 10 & 20 & 30 & 40 \\
$c_{a}$ & 50 & 100 & 100 & 150 \\
\hline
\end{tabular}


Table 2. Travel and demurrage cost

\begin{tabular}{ccccccccc}
\hline Port & A & B & C & D & E & F & G & H \\
\hline A & 3 & 3 & 4 & 6 & 4 & 8 & 7 & 3 \\
B & 3 & 4 & 3 & 3 & 7 & 3 & 4 & 3 \\
C & 4 & 3 & 2 & 5 & 2 & 2 & 3 & 4 \\
D & 6 & 3 & 5 & 3 & 5 & 2 & 3 & 7 \\
E & 4 & 7 & 2 & 5 & 1 & 3 & 2 & 3 \\
F & 8 & 3 & 2 & 2 & 3 & 2 & 5 & 5 \\
G & 7 & 4 & 3 & 3 & 2 & 5 & 4 & 3 \\
H & 3 & 3 & 4 & 7 & 3 & 5 & 3 & 2 \\
\hline
\end{tabular}

Table 3. Parameter values for each production ports

\begin{tabular}{ccccccccccc}
\hline \multirow{2}{*}{ Notation } & \multicolumn{2}{c}{ Port A } & \multicolumn{2}{c}{ Port B } & \multicolumn{2}{c}{ Port C } & \multicolumn{2}{c}{ Port D } & \multicolumn{2}{c}{ Port E } \\
\cline { 2 - 11 } & Oil-1 & Oil-2 & Oil-1 & Oil-2 & Oil-1 & Oil-2 & Oil-1 & Oil-2 & Oil-1 & Oil-2 \\
\hline$I P_{(j, 0)}$ & 2 & 2 & 3 & 3 & 4 & 4 & 3 & 3 & 5 & 5 \\
$I P_{(j, b, t)}^{\min }$ & 1 & 1 & 1 & 1 & 1 & 1 & 1 & 1 & 1 & 1 \\
$I P_{(j, b, t)}^{\max }$ & 100 & 100 & 100 & 100 & 100 & 100 & 100 & 100 & 100 & 100 \\
$f p_{j}^{\min }$ & 2 & 2 & 3 & 3 & 2 & 2 & 3 & 3 & 2 & 2 \\
$f p_{j}^{\max }$ & 15 & 15 & 10 & 10 & 12 & 12 & 14 & 14 & 13 & 13 \\
$D P_{v j t}^{I}$ & 10 & 10 & 15 & 15 & 12 & 12 & 20 & 20 & 17 & 17 \\
$D P_{v j t}^{O}$ & 15 & 15 & 16 & 16 & 18 & 18 & 19 & 19 & 20 & 20 \\
$m_{j}$ & 10 & 10 & 10 & 10 & 10 & 10 & 10 & 10 & 10 & 10 \\
\hline
\end{tabular}

Table 4. Parameter values for each consumption ports

\begin{tabular}{ccccccc}
\hline \multirow{2}{*}{ Notation } & \multicolumn{2}{c}{ Port F } & \multicolumn{2}{c}{ Port G } & \multicolumn{2}{c}{ Port H } \\
\cline { 2 - 7 } & Oil-1 & Oil-2 & Oil-1 & Oil-2 & Oil-1 & Oil-2 \\
\hline$I C_{(j, 0)}$ & 10 & 10 & 11 & 11 & 9 & 9 \\
$I C_{(k, b, t)}^{\min }$ & 1 & 1 & 1 & 1 & 1 & 1 \\
$I C_{(k, b, t)}^{\max }$ & 100 & 100 & 100 & 100 & 100 & 100 \\
$f c_{k}^{\min }$ & 0 & 0 & 0 & 0 & 0 & 0 \\
$f c_{k}^{\max }$ & 30 & 30 & 30 & 30 & 30 & 30 \\
$D C_{v k t}^{I}$ & 20 & 20 & 20 & 20 & 20 & 20 \\
$D C_{v k t}^{O}$ & 20 & 20 & 20 & 20 & 20 & 20 \\
$m_{k}$ & 10 & 10 & 10 & 10 & 10 & 10 \\
\hline
\end{tabular}

Table 5. Production and consumption level

\begin{tabular}{ccccccccccc}
\hline \multirow{2}{*}{ Port } & \multicolumn{2}{c}{$t=1$} & \multicolumn{2}{c}{$t=2$} & \multicolumn{2}{c}{$t=3$} & \multicolumn{2}{c}{$t=4$} & \multicolumn{2}{c}{$t=5$} \\
\cline { 2 - 11 } & Oil-1 & Oil-2 & Oil-1 & Oil-2 & Oil-1 & Oil-2 & Oil-1 & Oil-2 & Oil-1 & Oil-2 \\
\hline A & 10 & 10 & 15 & 15 & 15 & 15 & 12 & 12 & 11 & 11 \\
B & 12 & 12 & 12 & 12 & 11 & 11 & 12 & 12 & 14 & 14 \\
C & 11 & 11 & 14 & 14 & 12 & 12 & 12 & 12 & 3 & 3 \\
D & 11 & 11 & 13 & 13 & 14 & 14 & 12 & 12 & 15 & 15 \\
E & 11 & 11 & 12 & 12 & 11 & 11 & 11 & 11 & 11 & 11 \\
F & 3 & 3 & 5 & 2 & 3 & 5 & 1 & 4 & 3 & 2 \\
G & 4 & 3 & 2 & 4 & 4 & 3 & 2 & 4 & 3 & 5 \\
H & 3 & 2 & 1 & 4 & 3 & 2 & 2 & 2 & 2 & 1 \\
\hline
\end{tabular}


In the case of the distribution of this multiproduct, the total costs incurred were 733 and the vessels used / rented to deliver the product amounted to two vessels, Vessel 1 and Vessel 3. Both Vessel 1 and Vessel 3 transported two types of products at once. Ship 1 starts to be used when $t=1$ by visiting port $D$ to load two types of products at once with details $\mathrm{Oil}_{1}=6$ and $O i l_{2}=7$. After loading the product, Vessel 1 goes to port $\mathrm{F}$ to send the product to when $\mathrm{t}=2$, while Vessel 3 is only used / rented on the second day by visiting port $\mathrm{E}$ to load the product with details $O i l_{1}=5$ and $O i l_{2}=9$ and immediately sent to port $\mathrm{G}$ the next day. The ship travel route in this case can be seen in figure 2 .

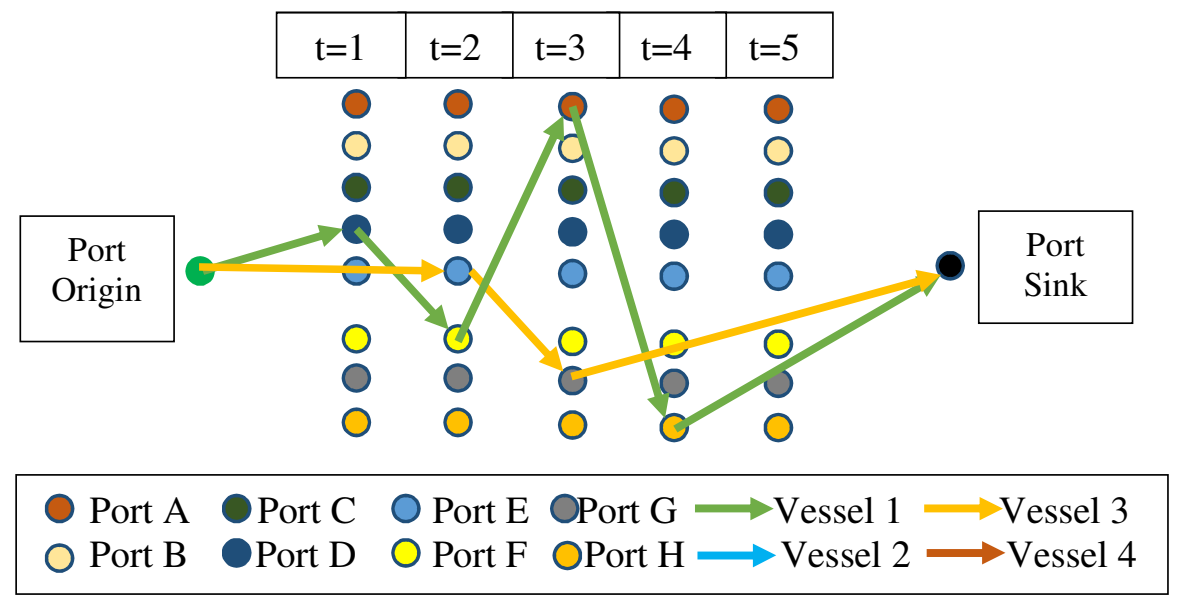

Figure 2. Vessel travel routes

\section{Conclusion}

In this scientific work it has been shown that the problem of determining the route of product distribution by ship, both when single and multi-products, can be seen as a mixed integer programming problem with an objective function to minimize ship operating costs. This fee consists of the cost of chartering the vessel and the overloading fee which exceeds the minimum part cargo of the vessel and is reduced by the return / netback of each delivery per product unit. Maritime Inventory Routing Model Problems in this scientific work both when multiproduct products for each optimization case were solved using the help of MiniZinc IDE 2.1.5 software with COIN-OR CBC (bundled) solver. The number of products shipped will be higher when the product return / netback value is greater than the excess charge per product unit, and the operational costs incurred by the company will be smaller. In the case of the distribution a single product can also use by this model by making set type of product sent is one.

\section{Acknowledgments}

Authors gratefully acknowledge financial support provided by Ministry of Research, Technology and Higher Education of the Republic of Indonesia under grant No. 011/SP2H/LT/DRPM/IV/2017 through the PUPT Scheme of Bogor Agricultural University.

\section{References}

[1] Agra, A., Christiansen, M., Hvattum, L.M., and Rodrigues, F. (2018). Robust optimization for a maritime inventory routing problem. Trans. Sci., Articles in Advance 1-17

[2] Al-Khayyal, F. and Hwang, S.J. (2007). Inventory constrained maritime routing and scheduling for multi-commodity liquid bulk, part I: applications and model. European Journal of Operational Research, 176: 106-130.

[3] Christiansen, M. (1999). Decomposition of a combined inventory and time constrained ship routing problem Transportation Science, 33(1): 3-16.

[4] Christiansen, M. and Fagerholt, K. (2009). Maritime inventory routing problem. In Encyclopedia of Optimization, Second Edition. Edited by Floudasand, C.A. and Pardalos, P.M. Springer, New York, 1947-1955. 
[5] Christiansen, M. and Nygreen, B. (1998). A method for solving ship routing problem with inventory constraints. Annals of Operations Research, 81: 357-378.

[6] Christiansen, M., Fagerholt, K., Flatberg, T., Haugen, Ø., Kloster, O., and Lund, E.H. (2011). Maritime inventory routing with multiple products: A case study from the cement industry. Euro. J. Oper. Res. 208: 86-94.

[7] Marriott, K., Stuckey, P.J., Koninck, L.D., and Samulowitz. (2014). A Minizinc Tutorial. Minizing.org.

[8] Papageorgiou, D.J., Nemhauser, G.L., Sokol, J., Cheon, M.S., Keha, A.B. (2014). MIRPLib - A library of maritime inventory routing problem instances: survey, core model, and benchmark results. Euro. J. Oper. Res. 235: 350-366.

[9] Song, J.H. and Furman, K.C. (2013). A maritime inventory routing problem: practical approach Comp. \& Ops. Res. 40: 657-665.

[10] UNCTAD. (2017). Review of Maritime Transport 2017. United Nations Publication, New York. 